\title{
Plasmids of Shigella flexneri serotype 1c strain Y394 provide advantages to bacteria in the host
}

\author{
Pawan Parajuli, Munazza I. Rajput and Naresh K. Verma* (B)
}

\begin{abstract}
Background: Shigella flexneri has an extremely complex genome with a significant number of virulence traits acquired by mobile genetic elements including bacteriophages and plasmids. S. flexneri serotype $1 c$ is an emerging etiological agent of bacillary dysentery in developing countries. In this study, the complete nucleotide sequence of two plasmids of S. flexneri serotype 1c strain Y394 was determined and analysed.

Results: The plasmid pINV-Y394 is an invasive or virulence plasmid of size 221,293 bp composed of a large number of insertion sequences (IS), virulence genes, regulatory and maintenance genes. Three hundred and twenty-eight open reading frames (ORFs) were identified in pINV-Y394, of which about a half (159 ORFs) were identified as IS elements. Ninety-seven ORFs were related to characterized genes (majority of which are associated with virulence and their regulons), and 72 ORFs were uncharacterized or hypothetical genes. The second plasmid pNV-Y394 is of size 10,866 bp and encodes genes conferring resistance against multiple antibiotics of clinical importance. The multidrug resistance gene cassette consists of tetracycline resistance gene tet $A$, streptomycin resistance gene strA-strB and sulfonamide-resistant dihydropteroate synthase gene sul2.
\end{abstract}

Conclusions: These two plasmids together play a key role in the fitness of Y394 in the host environment. The findings from this study indicate that the pathogenic $S$. flexneri is a highly niche adaptive pathogen which is able to co-evolve with its host and respond to the selection pressure in its environment.

Keywords: Shigella flexneri, Plasmids, Virulence, Multidrug resistance, Bacterial pathogen

\section{Background}

Shigella is a Gram-negative, non-motile, facultative anaerobic human enteric pathogen of the family Enterobacteriaceae that is closely related to Escherichia coli but has acquired specific traits of pathogenicity, physiology, and antigenic diversity [1]. The key to its virulence is the acquisition of a $210-230 \mathrm{~kb}$ virulence plasmid (VP) that enables the bacterium to invade and spread into the intestinal epithelial cells and induce apoptosis in the infected macrophages [2,3]. There are at least 190 million shigellosis cases and 70,000 deaths annually; principally in developing countries [4]. S. flexneri is the primary cause of shigellosis in the developing countries (up to $62 \%$ of all Shigella spp. infections) [5]. S. flexneri has no

\footnotetext{
* Correspondence: naresh.verma@anu.edu.au

Division of Biomedical Science and Biochemistry, Research School of Biology, The Australian National University, Canberra, ACT, Australia
}

less than 19 different serotypes based on their antigenic determinants present on the O-antigen of the outer membrane lipopolysaccharide (LPS) [6]. Out of which, $S$. flexneri serotype 1c has emerged as a significant serotype in developing countries over the last decade [7].

Mobile genetic elements including bacteriophages, insertion sequences and plasmids play a very important role in pathogen evolution and genomic plasticity in several bacterial pathogens and S. flexneri is not an exception. The VP of $S$. flexneri encodes essential virulence factors regulated by several genes located on the VP as well as on its chromosome [8]. The plasmid comprises a conserved pathogenicity island of $31 \mathrm{~kb}$ region which encodes the Ipa-Mxi-Spa type III secretion system (T3SS) [9]. While performing the complete genome sequencing of S. flexneri serotype 1c strain Y394 ("Y394" hereafter) [7], we recovered two plasmids which provide pathogenicity advantage to Y394 in the host environment. The

(c) The Author(s). 2019 Open Access This article is distributed under the terms of the Creative Commons Attribution 4.0 International License (http://creativecommons.org/licenses/by/4.0/), which permits unrestricted use, distribution, and 
first plasmid corresponds to the VP or invasion plasmid of Y394, hence named pINV-Y394. The second plasmid designated as pNV-Y394 possesses a multidrug-resistance cassette. This paper reports the complete nucleotide sequence and gene analysis of the two aforementioned plasmids to further facilitate the understanding of the evolution and pathogenic determinants of Y394, a newly emerged serotype, serotype 1c, of S. flexneri.

\section{Results}

\section{General features of pINV-Y394}

The large VP pINV-Y394 is of size 221,293 bp and comprises of 328 open reading frames (ORFs). The pINVY394 is a mosaic of essential virulence genes and their regulons, a significant number of IS elements and several hypothetical genes yet to be characterized (Fig. 1). The GC content of pINV-Y394 was found to be $45.9 \%$, which is lower than that of the Y394 chromosome (50.9\%).

The size and the composition, however, are similar to previously published S. flexneri serotype 2a VP, pCP301 (GenBank Accession number AL391753); S. flexneri serotype 5a VP, pWR100 (GenBank Accession number AF386526) and S. flexneri serotype 1a VP, unnamed plasmid1 (referred as "INV-0670" hereafter; GeneBank Accession number CP020087.1) (Table 1).

The pINV-Y394 has a similar arrangement of genes as seen in pCP301, pWR100 and INV-0670 with the exception of a large inversion and a few recombination events relative to the pINV-Y394 (Fig. 2). The $31 \mathrm{~kb}$ ipa-mxispa region is identical in all the compared VPs. We identified 27 unique genes in pINV-Y394 that are not present in three other compared plasmids (with 95\% identity cut-off). These genes code for IS elements/ transposases (21 ORFs), Osp2 domain protein (1 ORF), conjugation transfer protein (1 ORF) and uncharacterized or hypothetical proteins (4 ORFs).

\section{IS elements}

The sequence analysis of pINV-Y394 identified 159 ORFs related to IS elements, which accounts for $34 \%$ (75,492 bp of $221,293 \mathrm{bp}$ ) of the total plasmid sequence. These 159 IS elements belong to 36 different IS families with IS3 being most dominant (76 ORFs) and encode for 52 complete IS elements, 106 partial IS elements and 1 novel putative IS element (Table 2). These IS elements spread all over the genome and usually enclose the virulence-associated genes as well as the uncharacterized ORFs [Fig. 1] with potential virulence function owing to its low GC [10].

\section{Virulence associated genes}

The $31 \mathrm{~kb}$ region of ipa-mxi-spa genes lies at position 100,933 bp-132,292 bp of pINV-Y394. This region comprises of 39 coding sequences clustered together and is transcribed in two directions. The 12 genes from ipaJ to icsB are transcribed in one direction whereas 27 genes from ipgD to spaS/spa40 are in the opposite direction. The GC content of this region is $34.22 \%$ [range: $27 \%$ (ORF180; hypothetical gene)- 39\% (Spa47/IpaC)] which is lower than the overall GC content of the plasmid. The 39 ORFs include five ipa, six ipg, twelve $m x i$, nine spa, virB, ACP (Acyl Carrier Protein), ics $B$ and four uncharacterized genes. The ipa-mxi-spa region is flanked by truncated transposase on both the sides suggesting its origin from a single entity. The downstream to spaS lies a hypothetical gene (32\% GC) containing conserved protein domain family $Y m g B$. YmgB/AriR protein has been shown to be critical for biofilm formation and acid resistance in E. coli [11].

Besides the ipa-mxi-spa region, pINV-Y394 also possesses several other genes which might have a potential role in virulence. There are eleven putative osp genes (ospB, ospC1, ospC2, ospC3, ospD1, ospD2, ospD3, ospE1, $o s p E 2$, ospF, ospG) distributed across the plasmid genome. Several of these genes including ospB, ospC1, $o s p E 2$, and $o s p F$ have been shown to be regulated by the protein MxiE and is believed to have a role in post-invasion steps of infection [12].

Besides the invasion plasmid antigen (ipa) genes in the ipa-mxi-spa region, there are five more alleles of ipaH genes named as ipaH1.4, ipaH2.5, ipaH4.5, ipaH7.8, and ipaH9.8. These genes possess a novel E3 ligase (NEL) domain at the $\mathrm{C}$-terminus and a series of leucine-rich repeats at the $\mathrm{N}$-terminus which are found in many bacterial virulence factors. The leucine-rich repeats sequester a cysteine residue contained in the NEL domain until invasion has occurred allowing the release of NEL domain for site-specific function $[13,14]$.

The key regulatory gene for virulence function, virf, is located approximately $62 \mathrm{~kb}$ upstream of $\operatorname{vir} B$ of the ipa-mxi-spa locus. The activation of the plasmid-encoded regulatory genes requires environmental stimuli such as temperature, osmolarity, and changes in DNA supercoiling $[8,15,16]$. The VirF protein binds to the promotor of virB which acts as a transcriptional activator of the genes of the T3SS [17]. Approximately 12-kb downstream of spaS lies the virA and icsA/virG with a distance of $0.5-\mathrm{kb}$ between them. The product of these genes contributes to the intracellular spread and protection against autophagic degradation $[18,19]$. The other vir-gene, virK, which is located 43-kb downstream to virG is required for post-transcriptional control of the $\operatorname{virG}$ gene [20].

\section{Genes associated with plasmid maintenance and transfer functions}

The sequence analysis also identified at least five sets of genes related to toxin-antitoxin (TA) systems. This includes 


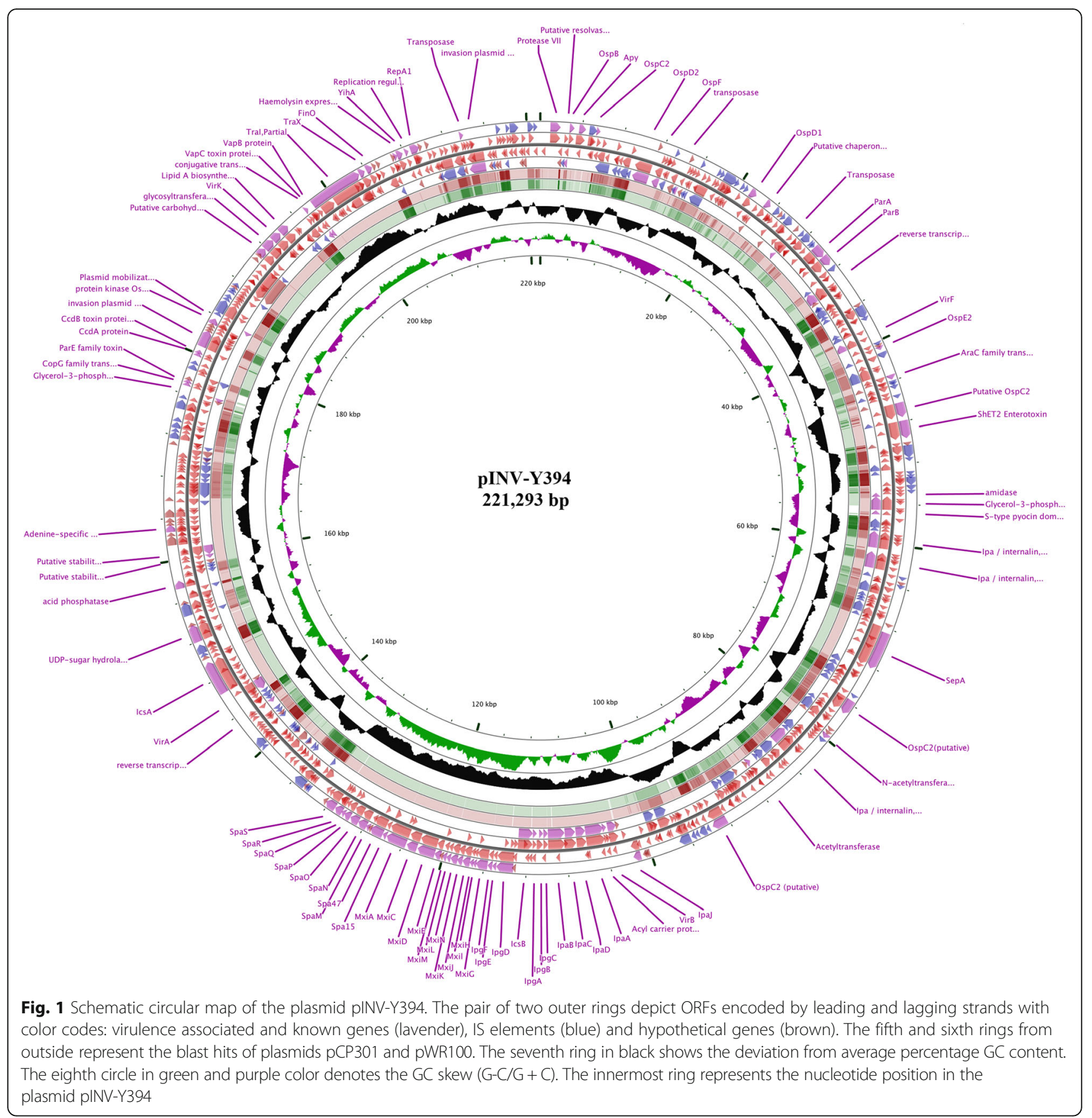

Table 1 General features of Shigella flexneri virulence plasmids

\begin{tabular}{llllllll}
\hline Plasmid & Host Strain & Plasmid Size (bp) & GC (\%) & ORFs Total & ORFs related to IS & ORFs Uncharacterized & Unique ORFs $^{a}$ \\
\hline pINV-Y394 & Shigella flexneri 1c strain Y394 & 221,293 & 45.9 & 328 & 159 & 72 & 27 \\
pCP301 & Shigella flexneri 2a strain 301 & 221,618 & 45.8 & 316 & 147 & 78 & 24 \\
pWR100 & Shigella flexneri 5a strain M90 T & 213,494 & 45.7 & 311 & 138 & 82 & 23 \\
unnamed1 & Shigella flexneri 1a strain 0670 & 228,834 & 46.1 & 301 & 145 & 93 & 35 \\
\hline
\end{tabular}

${ }^{a}$ Unique ORFs refer to ORFs which are present in corresponding plasmid only 


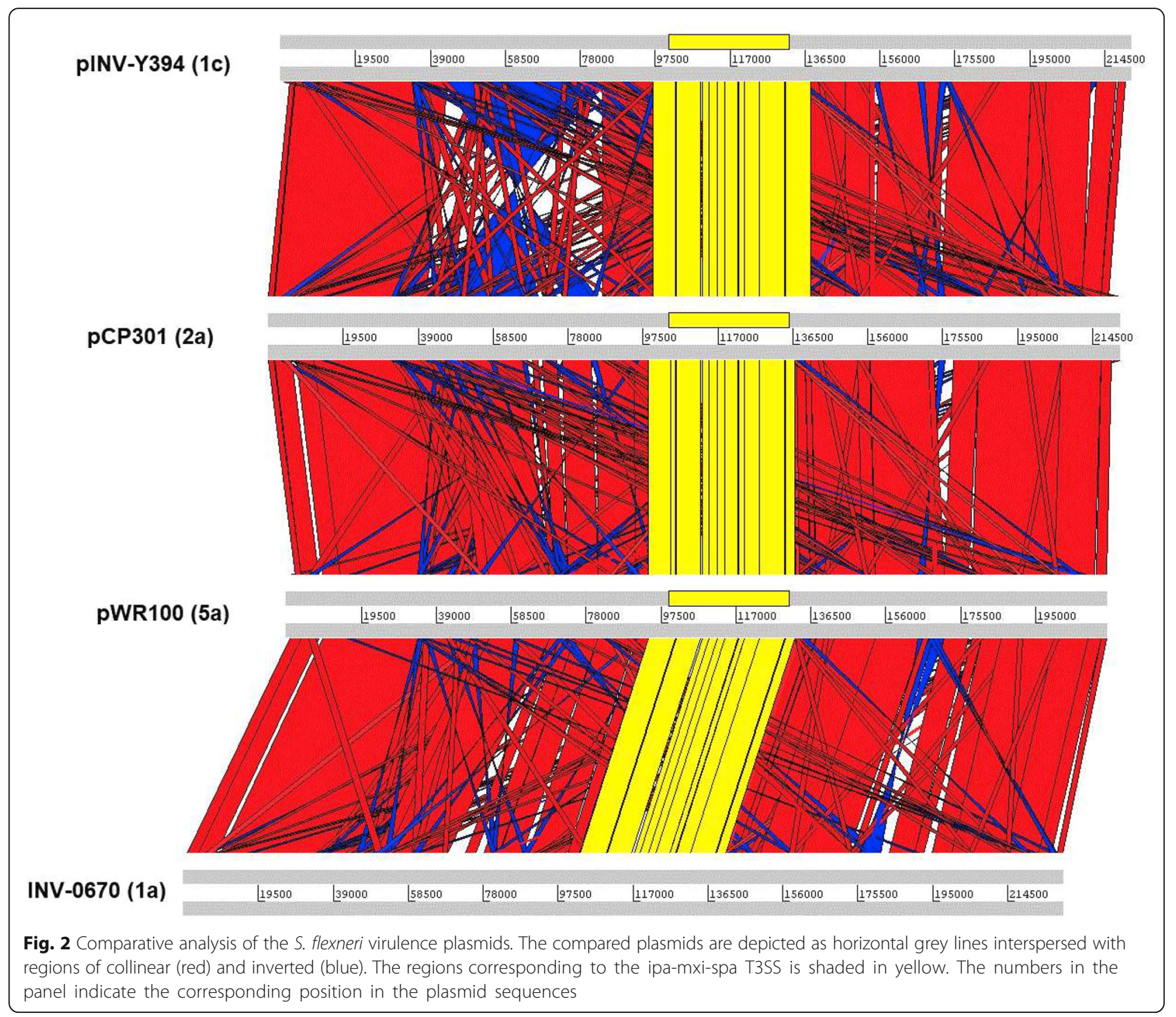

parAB, ccdAB, stbAB, vapBC and parE/copG. These genes are essential for maintaining the plasmids in bacteria through post-segregational killing mechanisms which eliminate bacterial cells lacking a plasmid after cell division. Besides, these genes are also involved in multiple cellular functions associated with survival under stress conditions [21].

pINV-Y394 consisted of several truncated conjugative transfer genes suggesting that it had conjugative transfer system but had lost its essential components over time. The plasmid possessed only $6 \%$ (45 AA) of the fully functional TraD (723 AA) which is an essential component of DNA transfer in bacterial conjugation system [22]. The pINV-Y394 lacks the essential region of TraD, the 38 amino acids at the very C-terminal end of the protein. The traI gene in PINV-Y394 has a deletion leading to a frameshift mutation. The two other genes present in PINV-Y394 related to conjugation are traX and $\operatorname{finO}$ which code for pilus acetylation protein and fertility inhibition protein. The FinO is one of the components of FinOP fertility inhibition complex. This protein inhibits the expression of traJ gene which in turn regulates the expression of some 20 other transfer genes. However, the remainder of the transfer genes was absent in pINV-Y394. The repeated conjugation experiments with pINV-Y394 were unsuccessful and are consistent with the previous observation [23].

\section{Plasmid pNV-Y394}

The plasmid pNV-Y394 is $10,866 \mathrm{bp}$ in size and has GC content of $61.54 \%$ which is well above the $51 \%$ GC content of the host bacteria Y394 (Fig. 3). The plasmid encodes multiple antibiotic resistance genes of clinical importance (Table 3). The plasmid is novel in S. flexneri but has partial homology of multidrug-resistance genes cassette (from nucleotides 5091 to 10,715) with other 
Table 2 Predicted Insertion Sequence (IS) family in pINV-Y394

\begin{tabular}{lllll}
\hline IS Family & Total ORFs & Complete IS & Different IS(s) & Total Size \\
\hline IS1 & 19 & 9 & 4 & 4806 \\
IS110 & 3 & 2 & 2 & 2568 \\
IS110 ssgr ${ }^{\text {I }}$ IS1111 & 2 & 0 & 2 & 1140 \\
IS21 & 4 & 2 & 1 & 4971 \\
IS3 ssgr IS2 & 11 & 3 & 4 & 4953 \\
IS3 ssgr IS3 & 32 & 11 & 4 & 11,718 \\
IS3 ssgr IS51 & 28 & 8 & 1 & 11,124 \\
IS3 ssgr IS150 & 5 & 1 & 3 & 1731 \\
IS4 ssgr IS4 & 3 & 3 & 1 & 3960 \\
IS4 ssgr IS10 & 2 & 1 & 1 & 1527 \\
IS4 ssgr IS50 & 1 & 0 & 1 & 1116 \\
IS5 ssgr IS427 & 7 & 3 & 1 & 2547 \\
IS66 & 18 & 8 & 4 & 12,699 \\
IS91 & 20 & 0 & 5 & 8376 \\
IS630 & 2 & 1 & 1 & 1617 \\
ISL3 & 2 & 0 & 1 & 639 \\
Total & 159 & 52 & 36 & 75,492 \\
\hline SSgr subgroup & & & &
\end{tabular}

${ }^{a_{\text {ssgr }} \text {-subgroups }}$

bacterial genomes and plasmids. The BLASTN result showed 100\% query cover and 99\% nucleotide identity with genomes and plasmids of several other clinical isolates including Salmonella enterica (GenBank accession numbers CP022498.1 and CP004059.1), Citrobacter freundii (GenBank accession number KY986974.1), Escherichia coli (GenBank accession number CP007137.1), Klebsiella pneumoniae (GenBank accession number CP021960.1) and Vibrio parahaemolyticus (GenBank accession number KY014465.1). The arrangement of genes in $12 \mathrm{~kb}$ region of the aforementioned genomes/plasmids along with $5 \mathrm{~kb}$ region of homology is shown in Fig. 4.

The multidrug cassette consists of genes sul2, strA-strB and tet $A$ which confers resistance against sulfonamide, streptomycin, and tetracycline, respectively. The phenotype was consistent with the genotype of pNV-Y394. The phenotypic expression of these antibiotic resistance genes was also confirmed with $E$. coli JM109 cells transformed with pNV-Y394.

Although pNV-Y394 harbored genes mobA and mobC associated with plasmid transfer and repA and repC associated with plasmid replication, we were unable to show the plasmid transfer by conjugation experiments, suggesting these genes are not sufficient for conjugative transfer.

\section{Discussion}

The large VP pINV-Y394 in S. flexneri serotype 1c strain Y394 encodes a 30-kb ipa-mxi-spa locus consisting of type III secretion system (T3SS) and an array of virulence factors which enable the bacteria to initiate invasion and subsequent interaction with the host epithelial cells and adapt intracellular lifestyle [2]. While the ipa, $m x i$ and spa genes are mostly involved in early stage of infection, the osp and ipaH genes are involved in post-invasion events [12]. The ipaH family genes are present on both the VP and the Shigella chromosome [24] and are capable of subverting the host's ubiquitination pathway [13, 14].

The low GC percentage of pINV-Y394 suggests that the pathogen has acquired the plasmid later as a response to adaptive evolution. There are a large number of IS elements in pINV-Y394 which accounts over one-third of the total plasmid genome. Most of the genes associated with virulence have lower GC content and are flanked by the IS elements suggesting that these genes have been brought as the result of numerous horizontal gene transfer via IS-mediated recombination events. The comparison of VP sequences from three other serotypes of S. flexneri showed a high level of similarity with some recombination and a large inversion $[23,25]$. The ipa-mxi-spa region was highly conserved in all the compared plasmids and is flanked by the IS elements at both the ends suggesting that these genes have a common origin and was acquired by IS-mediated recombination. Further, the few differences in gene content of the compared plasmids were mostly in the sequence of IS elements; suggesting all the compared plasmids had a common backbone. Out of 328 ORFs, about $26 \%$ of them are uncharacterized in PINV-Y394. Many of which have low GC content and flanked by IS elements, suggesting that these genes are foreign and might have brought to the plasmid by several IS-mediated recombination events. These uncharacterized genes warrant further studies on their function(s).

The lack of conjugative transfer of PINV-Y394 is consistent with previous studies [23, 25] and can be explained by the loss of intact tra locus which is essential for the exchange of plasmids between two bacteria by conjugation. The TraD protein which acts as a coupling protein and connects the DNA-processing machinery to the mating pair-forming transfer apparatus is truncated in PINV-Y394 with the loss of 38 amino acid C-terminal region essential for TraM interaction [22]. The genes traD, traM, and traY are involved in the oriT nicking, strand displacement, and DNA transfer during conjugation [26].

The other important gene of the tra locus, traI, has a frameshift mutation in its coding sequence. The protein TraI is a bifunctional protein which acts as a sequencespecific DNA trans-esterase, providing the site- and strand-specific nick required to initiate DNA transfer and a processive $5^{\prime}$ to $3^{\prime}$ helicase reaction that provides the motive force for strand transfer [27]. The loss of transfer function in VP has stabilized the virulence genes 


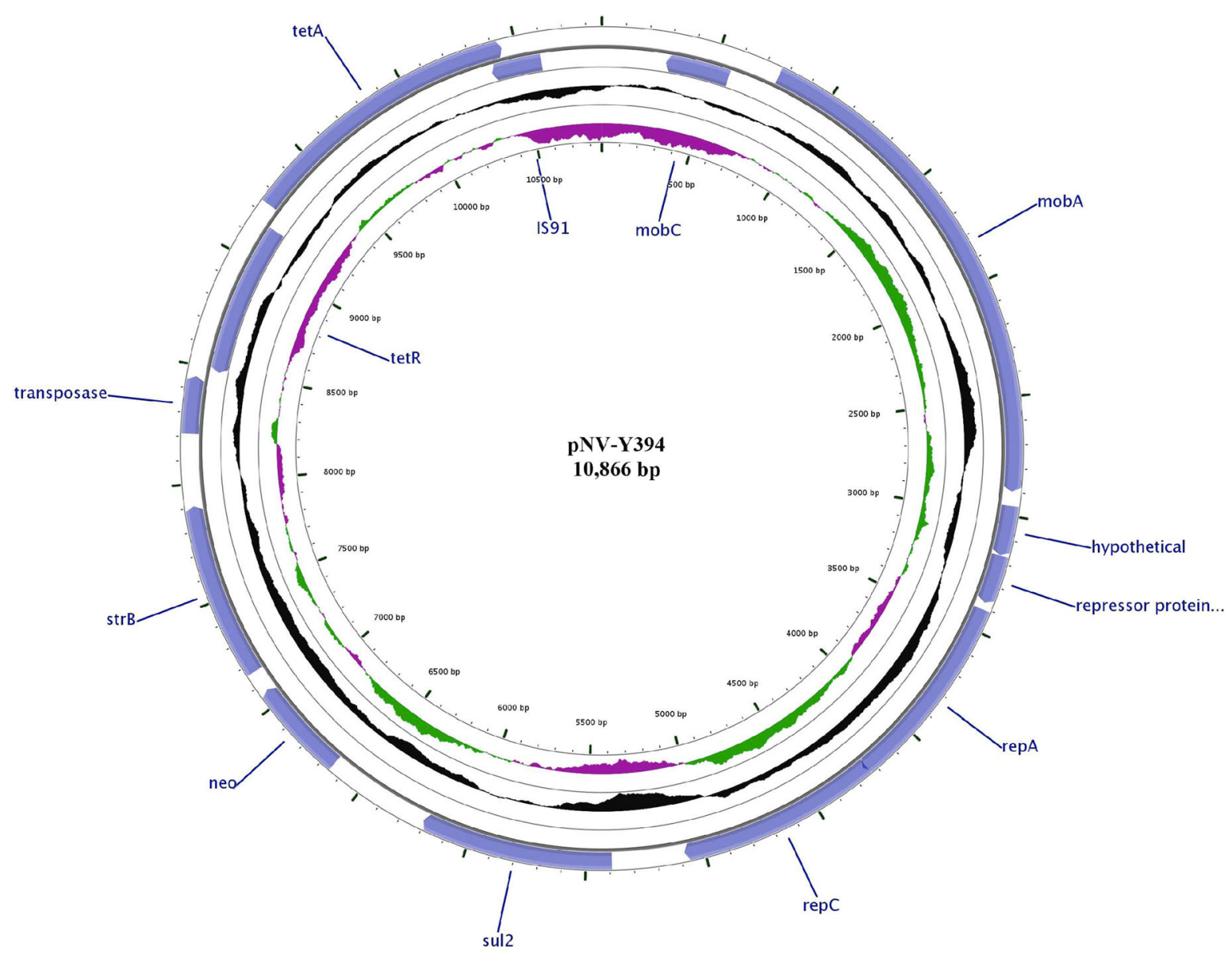

Fig. 3 Schematic circular map of the plasmid pNV-Y394. The two outer rings depict ORFs in pNV-Y394 (blue). The third ring in black shows the deviation from average percentage GC content. The fourth circle in green and purple color denotes the GC skew (G-C/G + C). The innermost ring represents the nucleotide position in the plasmid

Table 3 Features of pNV-Y394

\begin{tabular}{lllll}
\hline Feature/gene & Start & Stop & Length (bp) & Function \\
\hline mobC & 569 & 285 & 285 & Mobilization protein C \\
mobA & 768 & 2897 & 2130 & Conjugal transfer protein TraA \\
Hypothetical protein & 2958 & 3170 & 213 & Hypothetical protein (Multispecies of Bacteria) \\
Repressor protein F & 3172 & 3378 & 207 & Major facilitator superfamily MFS_1 \\
repA & 3408 & 4247 & 840 & IS, phage, Tn; Transposon-related functions \\
repC & 4234 & 5085 & 852 & Plasmid replication protein C \\
sul2 & 5393 & 6208 & 816 & Dihydropteroate synthase (EC 2.5.1.15) \\
strA/neo & 7672 & 420 & Aminoglycoside 3'-phosphotransferase (EC 2.7.1.95) \\
strB & 6653 & & Streptomycin 3'-kinase StrA (EC 2.7.1.87) \\
Putative transposase & 7164 & 7907 & Aminoglycoside 3'-phosphotransferase 2 (EC 2.7.1.95) \\
tetR & 8213 & 8455 & 243 & Streptomycin 3'-kinase StrB (EC 2.7.1.87) \\
tetA & 9164 & 8487 & 678 & Putative transposase/relaxase /helicase \\
IS91 & 9243 & 10,442 & 1200 & Transcriptional regulator, TetR family \\
\hline
\end{tabular}




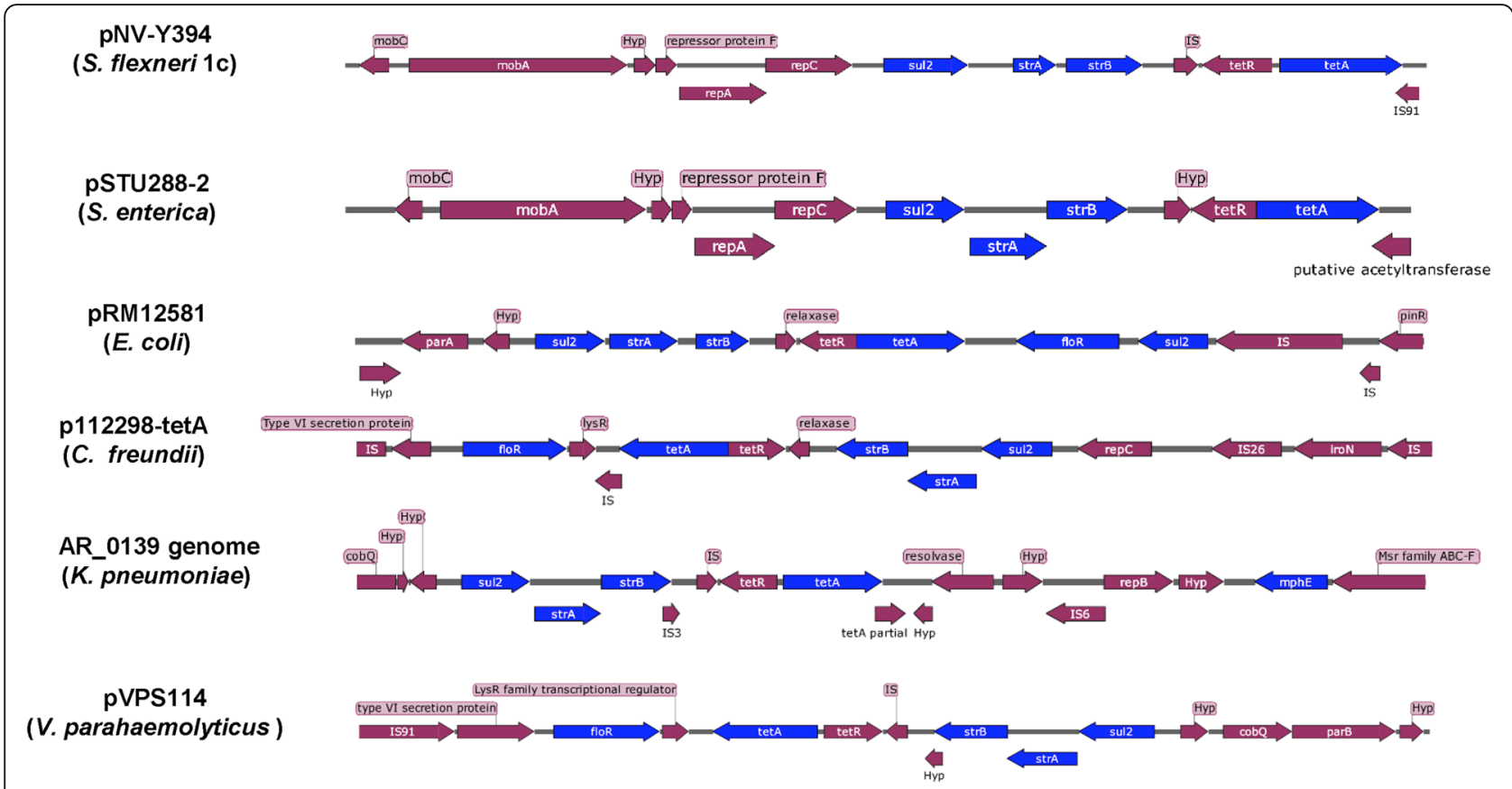

Fig. 4 Genomic organization of the plasmid pNV-Y394 and its homologues. The arrows indicate the direction of genes coded by forward and reverse strand on each of the compared plasmids/genome. The genes conferring drug resistance are higlighted in blue

alongside allowing parallel evolution of the VPs with the chromosomes [28].

Although, no other S. flexneri strain has been previously reported with multidrug resistance region as found in pNV-Y394, the 5-kb antibiotic resistance cassette of plasmid pNV-Y394 which provides resistance to the bacteria against sulfonamide, streptomycin and tetracycline antibiotics of clinical importance, has been isolated from several bacterial pathogens including S. sonnei [29], Salmonella enterica [30] and Klebsiella pneumoniae [31]. The antibiotic resistance cassette in pNV-Y394 is flanked by IS91 family transposases, which are linked to active rolling-circle transposition of several virulence and toxin genes and play an important role in pathogen evolution [32, 33]. Although, pNV-Y394 lacks complete conjugative transfer elements and is non-conjugative, the antibiotic gene cassette might have brought by IS91-mediated gene dissemination into Y394. The horizontal acquisition of multiple drug resistance genes has been suggested to play an important role in driving the global spread of a pathogen as a new epidemic clone $[34,35]$. The increase in multiple drug resistance strains of S. flexneri, endemic in developing countries, is of major concern due to limited resource settings for antibiotic susceptibility testing and limited therapeutic options. The antibiotic resistance surveillance studies across multiple Shigella endemic regions including China, India, Bangladesh, Pakistan, Nepal, Gabon, Kenya, Senegal, Iran and Egypt showed high percentage of S. flexneri isolates being resistant to tetracycline, trimethoprim-sulfamethoxazole, ampicillin and streptomycin [36-48]. This warrants the choice of antibiotic therapy be based on recent antibiotics susceptibility data of Shigella isolated from nearby geographical regions.

\section{Conclusions}

The comparison of pINV-Y394 with pCP301, pWR100 and INV-0670 showed that all four plasmids were highly conserved despite they were derived from different $S$. flexneri serotypes. This suggests that VP is a prerequisite for $S$. flexneri virulence and might have been acquired from a common ancestor. Further functional characterization of the conserved hypothetical genes would increase our understanding of virulence gene regulatory networks. The VP pINV-Y394 has the essential genetic element for its virulence and pNV-Y394 provides resistance to Y394 against an array of antibiotics of clinical importance. Hence, the two plasmids isolated from Y394 have an important role in the fitness of Y394 in the host environment. Furthermore, proper surveillance of antimicrobial resistance of this globally important pathogen is warranted, as this information is crucial for effective outbreak management and control of the disease.

\section{Methods}

Bacterial strains and plasmids

The two plasmids pINV-Y394 and pNV-Y394 presented in this study were derived from $S$. flexneri serotype 1c 
strain Y394 which was kindly provided to us by Nils I. A. Carlin [49]. The Y394 was grown aerobically (180 rpm) at $37^{\circ} \mathrm{C}$ in Luria Bertani broth (LB). The total bacterial DNA was prepared using the Genomic Tip 100/G (Qiagen) according to the manufacturer's instructions. The LB was supplemented with antibiotics (SigmaAldrich, Germany) where needed. The antibiotic susceptibility pattern of Y394 was determined as described previously [7].

\section{Plasmid DNA sequencing and assembly}

The complete genome of $S$. flexneri serotype 1c strain Y394 was sequenced using the hybrid method of single-molecule real-time (SMRT) sequencing technology and short-read MiSeq (Illumina) sequencing technology as described previously [7]. Briefly, The SMRTbell Template Prep Kit 1.0 (PacBio) was used for SMRT sequencing library preparation of about $20 \mathrm{~kb}$ insert size. The sequencing was performed using PacBio RSII sequencing system. The Nextera XT DNA library preparation kit (Illumina) was used for MiSeq v3 300bp paired-end sequencing. A de novo assembly of these reads was performed with HGAP.3 (Pacific Biosciences) on the SMRT Analysis Pipeline version 2.3.0 [50]. Following de novo assembly of the SMRT reads, a single contig of 4,584,634 bp (203X coverage) representing the Y394 chromosome was obtained (GenBank Accession number CP020753). The sequence reads that did not map to the chromosome were then independently assembled into two contigs of size 221,307 bp (250X coverage) representing pINV-Y394 and 10,873 bp (30X coverage) representing pNV-Y394. The circularization of the plasmid was obtained using a package Circlator [51]. To improve the quality of the SMRT sequences, the miseq reads were quality trimmed using Trimmomatic v0.36 [52] and used for error correction by using BWA-MEM and Pilon [53, 54]. After circularization and Pilon improvement the final size of pINV-Y394 was 221,293 bp and pNV-Y394 was 10,866 bp.

\section{DNA sequence annotation and analysis}

The automated annotation of plasmid sequences was performed using Rapid Annotation using Subsystem Technology (RAST) version 2.0 [55] followed by manual curation using BLASTP searches [56]. The IS elements were identified using ISsaga [57]. The antibiotic profiles of the plasmids were examined with ResFinder [58]. The genes that were common and unique in each of the compared plasmids were analysed using Roary [59].

The image files showing the synteny map of the plasmids were obtained using ACT (Artemis Comparison Tool) [60], CG View Server [61] and Snap Gene Viewer (Version 3.3.1).

\section{Plasmid characterization and tagging}

The plasmid pINV-Y394 was first tagged with kan gene for antibiotic selection using $\lambda$-red recombinase system as described previously [62]. Briefly, the Y394 strain was first transformed with pKD46 plasmid encoding $\lambda$-red recombinase system. Fifty-base pair overhang primers (Sigma, Australia) were designed corresponding to the putative reverse transcriptase gene of PINV-Y394 and used for amplification of kan gene using pKD4 miniprep DNA as a template (Additional file 1: Table S1). The PCR amplicons were then DpnI-treated; column purified and transformed with Y394+ pKD46 electrocompetent cells. The transformants were selected using LB agar plates containing kanamycin $(50 \mu \mathrm{g} / \mathrm{ml})$ and confirmed by Sanger sequencing.

To examine the phenotypic expression of antibiotics resistant genes in pNV-Y394, miniprep DNA was extracted using Wizard ${ }^{\bullet}$ Plus SV Minipreps DNA purification system (Promega, USA) and the plasmid preparations were used to transform electrocompetent Escherichia coli JM109 cells and the resultant clones were recovered using LB-agar plates containing either tetracycline $(10 \mu \mathrm{g} / \mathrm{ml})$ and/or streptomycin $(10 \mu \mathrm{g} / \mathrm{ml})$.

\section{Conjugation}

To determine the ability of conjugative transfer of the two plasmids, mating experiments were performed. The mating experiment was carried out by mixing the exponential growth phase cultures of donor S. flexneri serotype 1c strain Y394 and recipient E. coli JM109 transformed with pBCSK $(+)$ containing chloramphenicol-resistance gene $\left(\mathrm{Cm}^{r}\right)$ to aid in antibiotic selection. The mating mixture was incubated in a $37 \mathrm{C}$ water bath for $1 \mathrm{~h}$ and resuspended in PBS. The serial dilutions were then plated on LB plate containing either kanamycin $(50 \mu \mathrm{g} / \mathrm{ml})$ and chloramphenicol $(25 \mu \mathrm{g} / \mathrm{ml})$ or tetracycline $(10 \mu \mathrm{g} / \mathrm{ml})$ and chloramphenicol $(25 \mu \mathrm{g} / \mathrm{ml})$ to discriminate the conjugative transfer of plasmids pINV-Y394 and pNV-Y394, respectively.

\section{Additional file}

Additional file 1: Table S1. Primers used in this study. (PDF 89 kb)

\section{Abbreviations}

GC: Guanine and cytosine; IS: Insertion sequences; T3SS: Type III secretion system; VP: Virulence plasmid

\section{Acknowledgments \\ We thank Marcin Adamski for bioinformatics support and Nils I. A. Carlin for} providing S. flexneri serotype 1c strain Y394.

Funding

PP received an Endeavour PhD scholarship from the Australian Government, Department of Education and Training. The funders had no role in the study design, data collection, data analysis or writing of the manuscript. 
The corresponding author had full access to all the data in the study and final decision to submit for publication.

\section{Availability of data and materials}

The raw sequence reads and the complete genome sequence of pINV-Y394 and pNV-Y394 reported in this study has been deposited in GenBank under bioproject PRJNA382451 with complete genome sequence accession number CP030773 and CP030774 respectively.

\section{Authors' contributions}

PP and MR performed all the laboratory work. PP analysed the sequence data and prepared the figures and Tables. NV conceived and directed the study. PP and NV co-wrote the manuscript. All authors read and approved the final manuscript.

\section{Ethics approval and consent to participate}

Not applicable.

\section{Consent for publication}

Not applicable.

\section{Competing interests}

The authors declare that they have no competing interests.

\section{Publisher's Note}

Springer Nature remains neutral with regard to jurisdictional claims in published maps and institutional affiliations.

Received: 21 August 2018 Accepted: 11 April 2019

Published online: 29 April 2019

\section{References}

1. Killackey SA, Sorbara MT, Girardin SE. Cellular aspects of Shigella pathogenesis: focus on the manipulation of host cell processes. Front Cell Infect Microbiol. 2016;6:38.

2. Sansonetti PJ, Kopecko DJ, Formal SB. Involvement of a plasmid in the invasive ability of Shigella flexneri. Infect Immun. 1982;35(3):852-60.

3. Zychlinsky A, Prevost MC, Sansonetti PJ. Shigella flexneri induces apoptosis in infected macrophages. Nature. 1992;358(6382):167-9.

4. Pires SM, Fischer-Walker CL, Lanata CF, Devleesschauwer B, Hall AJ, Kirk MD, Duarte AS, Black RE, Angulo FJ. Aetiology-specific estimates of the global and regional incidence and mortality of Diarrhoeal diseases commonly transmitted through food. PLoS One. 2015;10(12):e0142927.

5. Gu B, Cao Y, Pan S, Zhuang L, Yu R, Peng Z, Qian H, Wei Y, Zhao L, Liu G, et al. Comparison of the prevalence and changing resistance to nalidixic acid and ciprofloxacin of Shigella between Europe-America and Asia-Africa from 1998 to 2009. Int J Antimicrob Agents. 2012;40(1):9-17.

6. Sun Q, Lan R, Wang J, Xia S, Wang Y, Wang Y, Jin D, Yu B, Knirel YA, Xu J. Identification and characterization of a novel Shigella flexneri serotype $Y v$ in China. PLoS One. 2013:8(7):e70238.

7. Parajuli P, Adamski M, Verma NK. Bacteriophages are the major drivers of Shigella flexneri serotype 1c genome plasticity: a complete genome analysis. BMC Genomics. 2017;18(1):722.

8. Maurelli AT, Sansonetti PJ. Identification of a chromosomal gene controlling temperature-regulated expression of Shigella virulence. Proc Natl Acad Sci U S A. 1988;85(8):2820-4

9. Sasakawa C, Kamata K, Sakai T, Makino S, Yamada M, Okada N, Yoshikawa M. Virulence-associated genetic regions comprising 31 kilobases of the 230kilobase plasmid in Shigella flexneri 2a. J Bacteriol. 1988;170(6):2480-4.

10. Lawrence JG, Ochman H. Amelioration of bacterial genomes: rates of change and exchange. J Mol Evol. 1997:44(4):383-97.

11. Lee J, Page R, García-Contreras R, Palermino J-M, Zhang X-S, Doshi O, Wood TK, Peti W. Structure and function of the Escherichia coli protein YmgB: a protein critical for biofilm formation and acid-resistance. J Mol Biol. 2007; 373(1):11-26.

12. Kane $C D$, Schuch R, Day WA Jr, Maurelli AT. MxiE regulates intracellular expression of factors secreted by the Shigella flexneri 2a type III secretion system. J Bacteriol. 2002;184(16):4409-19.

13. Quezada CM, Hicks SW, Galan JE, Stebbins CE. A family of Salmonella virulence factors functions as a distinct class of autoregulated E3 ubiquitin ligases. Proc Natl Acad Sci U S A. 2009;106(12):4864-9.
14. Rohde JR, Breitkreutz A, Chenal A, Sansonetti PJ, Parsot C. Type III secretion effectors of the IpaH family are E3 ubiquitin ligases. Cell Host Microbe. 2007 1(1):77-83.

15. Bernardini ML, Fontaine A, Sansonetti PJ. The two-component regulatory system ompR-envZ controls the virulence of Shigella flexneri. J Bacteriol. 1990;172(11):6274-81.

16. Dorman CJ, Ni Bhriain N, Higgins CF. DNA supercoiling and environmental regulation of virulence gene expression in Shigella flexneri. Nature. 1990; 344(6268):789-92

17. Adler B, Sasakawa C, Tobe T, Makino S, Komatsu K, Yoshikawa M. A dual transcriptional activation system for the $230 \mathrm{~kb}$ plasmid genes coding for virulence-associated antigens of Shigella flexneri. Mol Microbiol. 1989;3(5): 627-35.

18. Campbell-Valois FX, Sachse M, Sansonetti PJ, Parsot C. Escape of actively secreting Shigella flexneri from ATG8/LC3-positive vacuoles formed during cell-to-cell spread is facilitated by IcsB and VirA. MBio. 2015;6(3):e02567-14.

19. Ogawa M, Yoshimori T, Suzuki T, Sagara H, Mizushima N, Sasakawa C. Escape of intracellular Shigella from autophagy. Science. 2005;307(5710): 727-31

20. Nakata N, Sasakawa C, Okada N, Tobe T, Fukuda I, Suzuki T, Komatsu K, Yoshikawa M. Identification and characterization of virk, a virulenceassociated large plasmid gene essential for intercellular spreading of Shigella flexneri. Mol Microbiol. 1992;6(16):2387-95.

21. Fernandez-Garcia L, Blasco L, Lopez M, Bou G, Garcia-Contreras R, Wood T, Tomas M. Toxin-antitoxin Systems in Clinical Pathogens. Toxins (Basel). 2016;8(7).

22. Beranek A, Zettl M, Lorenzoni K, Schauer A, Manhart M, Koraimann G. Thirtyeight C-terminal amino acids of the coupling protein TraD of the F-like conjugative resistance plasmid $\mathrm{R} 1$ are required and sufficient to confer binding to the substrate selector protein TraM. J Bacteriol. 2004;186(20): 6999-7006.

23. Buchrieser C, Glaser P, Rusniok C, Nedjari H, D'Hauteville H, Kunst F, Sansonetti P, Parsot C. The virulence plasmid pWR100 and the repertoire of proteins secreted by the type III secretion apparatus of Shigella flexneri. Mol Microbiol. 2000;38(4): 760-71.

24. Venkatesan MM, Buysse JM, Hartman AB. Sequence variation in two ipaH genes of Shigella flexneri 5 and homology to the LRG-like family of proteins. Mol Microbiol. 1991;5(10):2435-45

25. Zhang J, Liu H, Zhang X, Yang J, Yang F, Yang G, Shen $Y$, Hou Y, Jin Q Complete DNA sequence and gene analysis of the virulence plasmid pCP301 of Shigella flexneri 2a. Sci China C Life Sci. 2003;46(5):513-21.

26. Raleigh EA, Low KB. Conjugation A2 - Maloy, Stanley. In: Hughes K, editor. Brenner's Encyclopedia of Genetics. 2nd ed. San Diego: Academic Press; 2013. p. 144-51.

27. Matson SW, Ragonese H. The F-plasmid Tral protein contains three functional domains required for conjugative DNA strand transfer. J Bacteriol. 2005;187(2): 697-706.

28. Yang J, Nie H, Chen L, Zhang X, Yang F, Xu X, Zhu Y, Yu J, Jin Q. Revisiting the molecular evolutionary history of Shigella spp. J Mol Evol. 2007;64(1):71-9.

29. Yang F, Yang J, Zhang $X$, Chen L, Jiang Y, Yan Y, Tang X, Wang J, Xiong Z, Dong J, et al. Genome dynamics and diversity of Shigella species, the etiologic agents of bacillary dysentery. Nucleic Acids Res. 2005:33(19): 6445-58.

30. Hooton SP, Timms AR, Cummings NJ, Moreton J, Wilson R, Connerton IF. The complete plasmid sequences of Salmonella enterica serovar typhimurium U288. Plasmid. 2014:76:32-9.

31. Sonnevend A, Yahfoufi N, Ghazawi A, Jamal W, Rotimi V, Pal T. Contribution of horizontal gene transfer to the emergence of VIM-4 carbapenemase producer Enterobacteriaceae in Kuwait. Infect Drug Resist. 2017;10:469-78.

32. del Pilar Garcillan-Barcia M, Bernales I, Mendiola MV, de la Cruz F. Singlestranded DNA intermediates in IS91 rolling-circle transposition. Mol Microbiol. 2001;39(2):494-501.

33. Garcillan-Barcia MP, de la Cruz F. Distribution of IS91 family insertion sequences in bacterial genomes: evolutionary implications. FEMS Microbiol Ecol. 2002:42(2):303-13.

34. Holt KE, Baker S, Weill FX, Holmes EC, Kitchen A, Yu J, Sangal V, Brown DJ, Coia JE, Kim DW, et al. Shigella sonnei genome sequencing and phylogenetic analysis indicate recent global dissemination from Europe. Nat Genet. 2012; 44(9):1056-9.

35. Holt KE, Wertheim H, Zadoks RN, Baker S, Whitehouse CA, Dance D, Jenney A, Connor TR, Hsu LY, Severin J, et al. Genomic analysis of diversity, population structure, virulence, and antimicrobial resistance in Klebsiella 
pneumoniae, an urgent threat to public health. Proc Natl Acad Sci U S A. 2015;112(27):E3574-81.

36. Soltan Dallal MM, Omidi S, Douraghi M, Haghi Ashtiani MT, Sharifi Yazdi MK, Okazi A. Molecular analysis of integrons and antimicrobial resistance profile in Shigella spp. isolated from acute pediatric diarrhea patients. GMS Hyg Infect Control. 2018;13:Doc02.

37. Afroze F, Ahmed T, Sarmin M, Smsb Shahid A, Shahunja KM, Shahrin L, Chisti MJ. Risk factors and outcome of Shigella encephalopathy in Bangladeshi children. PLoS Negl Trop Dis. 2017;11(4):e0005561.

38. Shakya G, Acharya J, Adhikari S, Rijal N. Shigellosis in Nepal: 13 years review of nationwide surveillance. J Health Popul Nutr. 2016;35(1):36.

39. Schaumburg F, Alabi AS, Kaba H, Lell B, Becker K, Grobusch MP, Kremsner PG, Mellmann A. Molecular characterization of Shigella spp. from patients in Gabon 2011-2013. Trans R Soc Trop Med Hyg. 2015;109(4):275-9.

40. Connor TR, Barker CR, Baker KS, Weill FX, Talukder KA, Smith AM, Baker S, Gouali M, Pham Thanh D, Jahan Azmi I, et al. Species-wide whole genome sequencing reveals historical global spread and recent local persistence in Shigella flexneri. Elife. 2015;4:e07335.

41. Zhang N, Lan R, Sun Q, Wang J, Wang Y, Zhang J, Yu D, Hu W, Hu S, Dai H, et al. Genomic portrait of the evolution and epidemic spread of a recently emerged multidrug-resistant Shigella flexneri clone in China. J Clin Microbiol. 2014:52(4):1119-26

42. Zhang J, Jin H, Hu J, Yuan Z, Shi W, Yang X, Xu X, Meng J. Antimicrobial resistance of Shigella spp. from humans in Shanghai, China, 2004-2011. Diagn Microbiol Infect Dis. 2014;78(3):282-6.

43. Sambe-Ba B, Seck A, Wane AA, Fall-Niang NK, Gassama-Sow A. Sensitivity to antibiotics and genetic support to resistance of Shigella flexneri strains isolated in Dakar from 2001 to 2010. Bull Soc Pathol Exot. 2013;106(2):89-94.

44. Njuguna HN, Cosmas L, Williamson J, Nyachieo D, Olack B, Ochieng JB, Wamola N, Oundo JO, Feikin DR, Mintz ED, et al. Use of population-based surveillance to define the high incidence of shigellosis in an urban slum in Nairobi, Kenya. PLoS One. 2013;8(3):e58437.

45. Das SK, Ahmed S, Ferdous F, Farzana FD, Chisti MJ, Latham JR, Talukder KA, Rahman M, Begum YA, Qadri F, et al. Etiological diversity of diarrhoeal disease in Bangladesh. J Infect Dev Ctries. 2013;7(12):900-9.

46. Ahmed SF, Klena J, Husain T, Monestersky J, Naguib A, Wasfy MO. Genetic characterization of antimicrobial resistance of Shigella flexneri 1c isolates from patients in Egypt and Pakistan. Ann Clin Microbiol Antimicrob. 2013;12:9.

47. Ghosh S, Pazhani GP, Chowdhury G, Guin S, Dutta S, Rajendran K, Bhattacharya MK, Takeda Y, Niyogi SK, Nair GB, et al. Genetic characteristics and changing antimicrobial resistance among Shigella spp. isolated from hospitalized diarrhoeal patients in Kolkata, India. J Med Microbiol. 2011;60(Pt 10:1460-6.

48. Wilson G, Easow JM, Mukhopadhyay C, Shivananda PG. Isolation \& antimicrobial susceptibility of Shigella from patients with acute gastroenteritis in Western Nepal. Indian J Med Res. 2006;123(2):145-50.

49. Wehler T, Carlin NI. Structural and immunochemical studies of the lipopolysaccharide from a new provisional serotype of Shigella flexneri. Eur J Biochem. 1988;176(2):471-6.

50. Chin CS, Alexander DH, Marks P, Klammer AA, Drake J, Heiner C, Clum A, Copeland A, Huddleston J, Eichler EE, et al. Nonhybrid, finished microbial genome assemblies from long-read SMRT sequencing data. Nat Methods. 2013;10(6):563-9.

51. Hunt M, Silva ND, Otto TD, Parkhill J, Keane JA, Harris SR. Circlator: automated circularization of genome assemblies using long sequencing reads. Genome Biol. 2015;16(1):294.

52. Bolger AM, Lohse M, Usadel B. Trimmomatic: a flexible trimmer for Illumina sequence data. Bioinformatics. 2014;30(15):2114-20.

53. Li H, Durbin R. Fast and accurate long-read alignment with burrows-wheeler transform. Bioinformatics. 2010;26(5):589-95.

54. Walker BJ, Abeel T, Shea T, Priest M, Abouelliel A, Sakthikumar S, Cuomo CA, Zeng Q, Wortman J, Young SK, et al. Pilon: an integrated tool for comprehensive microbial variant detection and genome assembly improvement. PLoS One. 2014:9(11):e112963.

55. Aziz RK, Bartels D, Best AA, DeJongh M, Disz T, Edwards RA, Formsma K, Gerdes S, Glass EM, Kubal M, et al. The RAST server: rapid annotations using subsystems technology. BMC Genomics. 2008;9:75.

56. Altschul SF, Gish W, Miller W, Myers EW, Lipman DJ. Basic local alignment search tool. J Mol Biol. 1990;215(3):403-10.

57. Varani AM, Siguier P, Gourbeyre E, Charneau V, Chandler M. ISsaga is an ensemble of web-based methods for high throughput identification and semi-automatic annotation of insertion sequences in prokaryotic genomes. Genome Biol. 2011;12(3):R30

58. Zankari E, Hasman H, Cosentino S, Vestergaard M, Rasmussen S, Lund O, Aarestrup FM, Larsen MV. Identification of acquired antimicrobial resistance genes. J Antimicrob Chemother. 2012;67(11):2640-4.

59. Page AJ, Cummins CA, Hunt M, Wong VK, Reuter S, Holden MT, Fookes M, Falush D, Keane JA, Parkhill J. Roary: rapid large-scale prokaryote pan genome analysis. Bioinformatics. 2015;31(22):3691-3.

60. Carver TJ, Rutherford KM, Berriman M, Rajandream MA, Barrell BG, Parkhill J. ACT: the Artemis comparison tool. Bioinformatics. 2005;21(16):3422-3.

61. Grant JR, Stothard P. The CGView server: a comparative genomics tool for circular genomes. Nucleic Acids Res. 2008;36(Web Server):W181-4.

62. Datsenko KA, Wanner BL. One-step inactivation of chromosomal genes in Escherichia coli K-12 using PCR products. Proc Natl Acad Sci U S A. 2000; 97(12):6640-5.
Ready to submit your research? Choose BMC and benefit from:

- fast, convenient online submission

- thorough peer review by experienced researchers in your field

- rapid publication on acceptance

- support for research data, including large and complex data types

- gold Open Access which fosters wider collaboration and increased citations

- maximum visibility for your research: over $100 \mathrm{M}$ website views per year

At BMC, research is always in progress.

Learn more biomedcentral.com/submissions 